\title{
Lebensgefährliche Manipulation
}

\section{Soldat überlistete}

\section{Schmerzpumpe}

Ärzte des AKH Linz berichten über zwei Fälle von massiver Opioidüberdosierung bei der intravenösen patientenkontrollierten Analgesie (PCA). Was zunächst wie ein Gerätedefekt aussah, erwies sich als missbräuchliche Anwendung der Pumpe durch die Patienten.

Die I.v.-PCA hat sich heute als Standard der parenteralen postoperativen Schmerztherapie etabliert. Wenn die kontinuierliche Schmerzmittelzufuhr nicht ausreicht, kann der Patient - ohne Arzt oder Pflegepersonal einschalten zu müssen - zusätzliche Boli abfordern. In Studien und im Alltag hat sich diese Therapieform als sicher und effizient erwiesen. Auf ein bisher wenig beachtetes Risiko machen Dr. Karin Imhof und Kollegen vom AKH Linz in der Zeitschrift „Der Anaesthesist“ aufmerksam: Zwei traumatisierte Patienten, die im Krankenhaus behandelt wurden, hatten ihre PCA-Pumpe gezielt manipuliert, um höhere Opioiddosen abrufen zu können.

\section{Missbräuchlicher Einsatz des Arztbolus}

Beide Patienten waren in Kriegsgebieten verletzt und zunächst dort versorgt worden. Patient 1 wurde mit chronischem Hüftgelenkempyem und Hüftkopfnekrose links eingeliefert. Die Schmerztherapie bestand zunächst aus Pregabalin und Dihydrocodein sowie Paracetamol und Metamizol bei Bedarf. Nach der Resektion des linken Hüftkopfes erhielt der Patient eine I.v.-PCA mit Hydromorphon (Basistherapie: 0,2 mg/h, Bolus: 0,2 mg mit einer Sperrzeit von 15 Minuten). Weil die Schmerzkontrolle unzureichend war, wurde nach einer Woche zusätzlich eine patientenkontrollierte epidurale Analgesie (PCEA) mit Naropin und Fentanyl installiert. Wegen Entzündung der Einstichstelle musste der Epiduralkatheter aber nach einer Woche wieder entfernt werden. Danach wurde bei der I.v.-PCA erstmals ein durch die Pumpeneinstellung nicht erklärbarer Anstieg des Tagesverbrauchs von 9,3 auf 19,3 mg Hydromorphon beobachtet.
Da man einen Pumpendefekt annahm, wurde zunächst nur das Gerät ausgetauscht. Erst als erneut Diskrepanzen zwischen angezeigter Tagesdosis und aufgezeichneten Bolusgaben auftraten, ging man der Sache auf den Grund: Wie dem Ereignisprotokoll zu entnehmen war, hatte der Patient den Arztbolus abgerufen und sich innerhalb von 24 Stunden insgesamt $36,5 \mathrm{mg}$ (!) Hydromorphon zugeführt.

Der Arztbolus ist ein zusätzlicher unlimitierter Schmerzmittelbolus, der durch einen Sicherheitscode geschützt ist. Offenbar war es dem Patienten jedoch gelungen, den Code zu knacken. Die nachfolgende Therapieumstellung auf transdermales Fentanyl und Hydromorphon bei Bedarf führte zu einer so schweren Entzugssymptomatik, dass der Patient fünf Tage lang auf die Intensivstation verlegt wurde.

Patient 2 wurde wegen eines Hautweichteildefekts nach rechtsseitiger Oberschenkelamputation behandelt. Er wurde postoperativ mit einer I.v.-PCA mit Hydromorphon und später zusätzlich mit einer PCEA versorgt. Auch bei ihm kam es nach Entfernung der PCEA zu einer missbräuchlichen Anwendung der I.v.-PCA durch Abforderung des Arztbolus.

\section{Den Code gab es im Internet}

Wie aber waren die Patienten an den Code für den Arztbolus gekommen? In den beiden Fällen waren Pumpen mit einem dreistelligen Verriegelungscode verwendet worden. „Ein gewisses Risiko, dass die Patienten durch Ausprobieren verschiedener Kombinationen oder durch Zufall den Code entschlüsseln, ist damit immer gegeben“, konstatieren Imhof und Kollegen. „Überraschend“ war für die Linzer Ärzte jedoch die Erkenntnis, dass der Code im

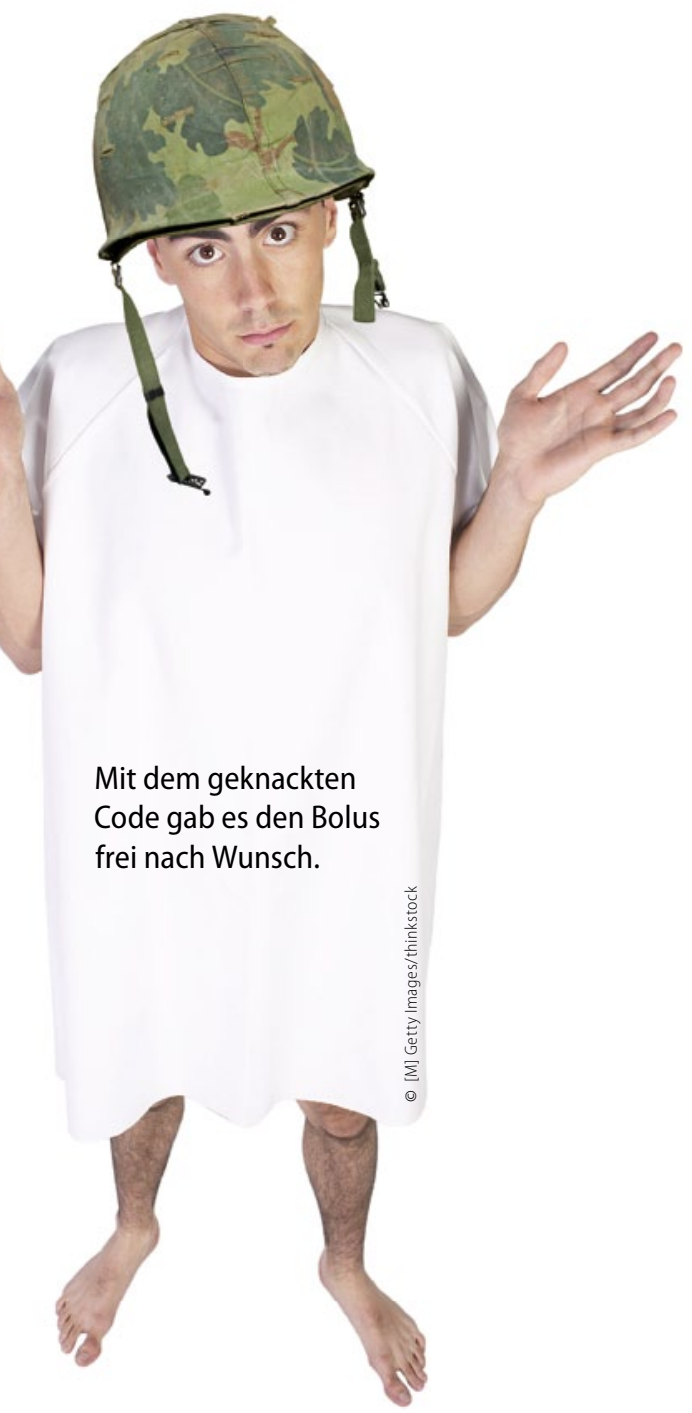

Internet problemlos zu finden war. Binnen 30 min. konnten die Codes von sechs der neun am häufigsten verwendeten Pumpen ausfindig gemacht werden. Das Hauptproblem besteht aber darin, dass bei fünf der neun Geräte der Arztboluscode zudem nicht umprogrammierbar war.

Um das Missbrauchsrisiko möglichst klein zu halten, seien geschultes Personal, tägliche Schmerzvisiten und eine standardisierte Einstellung der Schmerzpumpen mit standardisierten Medikamentenkonzentrationen erforderlich. „Außerdem sollten nur Geräte mit veränderbaren Codes eingesetzt und diese in regelmäßigen Abständen umprogrammiert werden.“

(Beateschumacher) 